\title{
ANÁLISIS MORFOMÉTRICO DE LAS ESPECIES DE DIPTERYX EN LA AMAZONÍA PERUANA
}

David Roy ALDANA GOMERO ${ }^{1}$, Carmen Rosa GARCÍA-DÁVILA², Carlos Gabriel HIDALGO PIZANGO $^{2}$, Gerardo Rafael FLORES LLAMPAZO ${ }^{2}$, Dennis DEL CASTILLO TORRES ${ }^{2}$, Carlos REYNEL RODRIGUEZ ${ }^{1}$, Eli PARIENTE MONDRAGÓN ${ }^{1}$, Eurídice Nora HONORIO CORONADO ${ }^{2}$

1 Universidad Nacional Agraria La Molina, Av. La Molina s.n., Lima, Perú

2 Instituto de Investigaciones de la Amazonía Peruana, Av. Abelardo Quiñones km 2.5, Iquitos, Perú. Correspondencia: E. Honorioeurihc@yahoo.com

\section{RESUMEN}

Las especies de Dipteryx en el Perú son reconocidas en campo por los pobladores locales y materos bajo el nombre de Shihuahuaco. Esto determina que la creciente demanda en la extracción de su madera para pisos y exteriores en la industria forestal se realice sin conocer las especies botánicas involucradas. El objetivo del presente estudio fue determinar las características morfológicas que diferencian las especies de Dipteryx en el Perú mediante el uso de análisis multivariados. Para esto, realizamos mediciones de 30 caracteres morfológicos foliares en 74 especímenes colectados de árboles provenientes de bosques naturales y muestras botánicas recopiladas de herbarios, así como mediciones de cinco caracteres morfológicos florales en siete especímenes fértiles disponibles. Los resultados muestran que existen tres grupos morfológicos que constituyen taxones con características consistentes y con distribución geográfica particular. D. micrantha morfotipo 1 con 3 a 5 pares de foliolos, foliolos de $5.4 \mathrm{~cm}$ x $11.6 \mathrm{~cm}$ en promedio, amplia distribución en la Amazonía baja, colectada desde el sur del departamento de Loreto hasta Madre de Dios. D. micrantha morfotipo 2 con 4 a 6 pares de foliolos, foliolos $3.0 \mathrm{~cm}$ x $7.4 \mathrm{~cm}$ en promedio, amplia distribución, colectada en Loreto y Ucayali. D. charapilla con 3 a 4 pares de foliolos, foliolos de $6.8 \mathrm{~cm}$ x $14.4 \mathrm{~cm}$, distribución restringida a unas pocas localidades en Loreto. La descripción de las especies y los mapas de distribución obtenidos en este estudio son insumos importantes para los censos forestales, el desarrollo de los planes de manejo forestal y la toma de decisión sobre la conservación de las especies de Dipteryx en la Amazonía peruana.

PALABRAS CLAVES: Análisis discriminante lineal, Coumarouna, PCA, shihuahuaco, UPGMA

\section{MORPHOMETRIC ANALYSIS OF THE SPECIES OF DIPTERYX IN THE PERUVIAN AMAZON}

\begin{abstract}
The species of Dipteryx in Peru are recognized in the field by the local and native inhabitants under the name of Shihuahuaco. This determines that the growing demand in the extraction of its wood for floors and exteriors in the forest industry is carried out without knowing the botanical species involved. The objective of the present study was to determine the morphological characteristics that differentiate Dipteryx species in Peru through the use of multivariate analyzes. For this, we measured 30 foliar morphological characters in 74 specimens of tree individuals collected from natural forests and of botanical samples compiled from herbaria, as well as, five floral characters measured in seven fertile available specimens. The results showed that there are three taxonomic groups that constitute morphologically consistent taxa with distinct geographic distributions. D. micrantha morphotype 1 with 3-5 pairs of leaflets, leaflets of $5.4 \mathrm{~cm} \times 11.6 \mathrm{~cm}$ on average, wide distribution in the lowland Amazon, collected from the south of the department of Loreto to Madre de Dios. D. micrantha morphotype 2 with 4-6 pairs of folioles, leaflets $3.0 \mathrm{~cm} \times 7.4 \mathrm{~cm}$ on average, wide distribution, collected in Loreto and Ucayali. D. charapilla with 3-4 pairs of leaflets, leaflets of $6.8 \mathrm{~cm} \times 14.4$ $\mathrm{cm}$, distribution restricted to a few localities in Loreto. The species descriptions and distribution maps obtained in this study are important inputs for the forest censuses, the development of the forest management plans and the decision making on the conservation of the Dipteryx species in the Peruvian Amazon.
\end{abstract}

KEYWORDS: Linear discriminant analysis, Coumarouna, PCA, shihuahuaco, UPGMA 


\section{INTRODUCCIÓN}

Dipteryx Scherb. (Fabaceae) es un género neotropical, ocurre en América del Sur, América Central y el Caribe, y presenta 12 especies generalmente de árboles emergentes (Barham, 2005). Las especies de Dipteryx en Perú son principalmente apreciadas como madera en la industria forestal debido a su dureza, ideal para pisos y aplicaciones externas (Reynel et al., 2003). Según los registros reportados en Perú Forestal en Números se ha extraído cerca de 900 mil metros cúbicos de madera de Dipteryx en el periodo 20102014, con una creciente demanda en las exportaciones principalmente dirigidas a la China (Putzel, 2010). Como para la mayoría de las especies maderables en Perú, monitorear la extracción y asegurar el manejo sostenible de las especies forestales es un desafío. Un factor limitante clave para el manejo sostenible de Dipteryx en Perú es la presencia de especies con delimitación morfológica poco clara. Apenas existe un intento de actualizar la situación taxonómica de las especies de Dipteryx (de Lima, 1989) desde la última revisión del género realizada a fines de los 40s (Ducke, 1949). Esta falta de conocimiento taxonómico básico significa que los rangos de distribución de las especies no están claros, su abundancia y ecología no pueden ser fácilmente evaluadas, y es difícil diseñar estrategias de manejo viables y efectivas. Por lo tanto, existe una necesidad de mejorar el conocimiento de la taxonomía y distribución de las especies de Dipteryx en Perú.

En el Perú, se ha registrado la presencia de al menos seis especies de Dipteryx, cuatro especies citadas en Flora del Perú bajo el género Coumarouna Aublet que actualmente es el sinónimo de Dipteryx (Macbride, 1943) [1. D. charapilla (J.F. Macbr.) Ducke, 2. D. ferrea (Ducke) Ducke, 3. D. micrantha Harms, 4. D. odorata (Aubl.) Willd.], y dos especies adicionales agregadas en el listado de especies de angiospermas y gimnospermas de Perú (Brako \& Zarucchi, 1993) [5. D. alata Vogel, 6. D. rosea Spruce ex Benth.]. Los registros de herbarios muestran que la mayoría de estas especies ocurren en el departamento de Loreto (Spichiger et al., 1989; Vasquez, 1997; Brako \& Zarucchi, 1993), mientras D. alata es reportada para Huánuco y Ucayali (Brako \& Zarucchi, 1993; Monteagudo y Huamán, 2010; Monteagudo et al., 2015). Sin embargo, en campo aparentemente no es fácil determinar estas especies, por lo que la comercialización y exportación de la madera se lleva a cabo bajo el nombre de Coumarouna odorata o Dipteryx spp ("shihuahuaco" o "cumaru"). Poder diferenciar las especies usando características morfológicas sería de ayuda en los inventarios y en la supervisión de las concesiones forestales en el Perú (Dávila et al., 2008). Más aún, conocer el rango de distribución geográfico fomentaría la aplicación de estudios genéticos a nivel de especies y de poblaciones, pudiendo así aplicar herramientas genéticas complementarias a la taxonomía tradicional para la verificación de la procedencia de la madera (Degen et al., 2013).

La morfología y morfometría foliar en las plantas varía entre las diferentes especies y dentro de una misma especie, es así que a través del uso de estudios morfométricos utilizando los análisis multivariados se pueden resumir las variables evaluadas con el fin de determinar las características morfológicas importantes que segregan a las especies de grupos taxonómicos de interés (Hammer et al., 2001). Estos estudios en especies forestales han sido usados para comparar la variación morfológica entre especies de un mismo género que son muy similares entre sí. Este es el caso del Quercus sartorii y Q. xalapensis realizado por Martinéz-Cabrera et al. (2011) que demuestra que los caracteres cuantitativos como el patrón de venación, la forma y número de areolas, entre otros, contribuyeron a la distinción de cada especie. Además, el estudio morfométrico del género Indigofera (Fabaceae) realizado por Soladoye et al. (2010) menciona que los caracteres foliares como ancho y largo de los foliolos son los que contribuyen significativamente a la delimitación de las diferentes especies de este género. En el caso de Hymenaea courbaril (Fabaceae) se trabajó al nivel de variedades, quedando sus seis variedades agrupadas en tres especies y en donde el análisis morfométrico se realizó junto con información geográfica y de hábitat (Mascarenhas et al., 2014). Estos estudios de las variaciones morfológicas foliares han sido una importante herramienta para entender la diferenciación fenotípica entre especies complejas y poder detectar las características morfológicas importantes que ayuden en los inventarios rápidos donde rara vez se cuenta con material fértil.

El objetivo del presente estudio fue contribuir al conocimiento taxonómico y dendrológico del género Dipteryx en Perú. Para esto, utilizamos análisis multivariados para identificar las características morfológicas que diferencian las especies de Dipteryx; estas mediciones fueron tomadas de especímenes colectados de árboles provenientes de bosques naturales y muestras botánicas recopiladas de herbarios con el fin de contestar las siguientes preguntas: (1) ¿Cuáles son los caracteres morfológicos que diferencian las especies de Dipteryx en Perú?, (2) ¿Cuál es la 
distribución geográfica de las especies de Dipteryx caracterizadas morfológicamente?, y (3) ¿Es Dipteryx odorata la especie más comercializada en Perú?. Este estudio dará las bases para entender mejor la ecología, delimitar la distribución de las especies y desarrollar óptimos planes de manejo y conservación de las especies de Dipteryx en la Amazonía peruana.

\section{MATERIAL Y MÉTODOS}

\section{EL GÉNERO DIPTERYX}

El género Dipteryx se caracteriza por presentar raíces tabulares, hojas compuestas, alternas, paripinnadas o aparentemente imparipinnadas, y raquis alado y prolongado en el ápice. Las inflorescencias se presentan en panículas terminales; los frutos son drupas indehiscentes, carnosas, ovoides, aceitosas; y las semillas son aromáticas (Vásquez, 1997). Dentro de la familia Fabaceae, los géneros de la tribu Dipterygeae, a la cual pertenecen Dipteryx, Taralea y Pterodon se caracterizan por tener flores con cáliz dividido en dos lóbulos, corola con pétalos diferenciados en estandarte, quilla y alas (Cardoso et al., 2015). Dipteryx suele ser confundido con otros géneros de la familia, como con Taralea por sus hojas gruesas y raquis alado, con Apuleia por la presencia de raíces tabulares amplias y con Andira por sus frutos (Macbride, 1892; Francisco, 2010).

\section{CARACTERES MORFOLÓGICOS}

Examinamos los caracteres morfológicos de 74 especímenes de herbario del género Dipteryx colectados en Perú (Figura 1), 56 colectados para el presente estudio (depositados en el Herbario Herrerense - HH) y 18 recopilados de herbarios digitales (MOL, US, RB, BRIT, F). Los especímenes colectados fueron digitalizados para su posterior análisis usando una cámara digital NIKON D800. La morfología de las hojas fue analizada usando 30 caracteres morfológicos vegetativos, de los cuales 24 son variables cuantitativas y seis son variables cualitativas (Tabla 1). Las variables cuantitativas incluyen el tamaño del foliolo (largo y ancho), de la base, del peciolulo, ápice, raquis, distancia entre foliolos, número de foliolos por hoja, desplazamiento de la vena media, ángulo de base y las proporciones entre las variables. Las mediciones fueron tomadas en 3 a 4 foliolos maduros por espécimen, usando una regla con $1 \mathrm{~mm}$ de precisión en el programa Image J (Schneider et al., 2012). Todas las variables cuantitativas fueron promediadas por espécimen, obteniendo una matriz general de datos. Las variables cuantitativas continuas como las longitudes fueron multiplicadas por 10 y sumadas más 1 en el caso de valores pequeños, y las proporciones fueron multiplicadas por 100. Estas variables y los ángulos se transformaron con logaritmo base $10\left(\log _{10}\right)$ y la variable cuantitativa discreta (número de foliolos) fue transformada usando la raíz cuadrada $(\sqrt{ } \mathrm{x})$. Las seis variables cualitativas fueron utilizadas para complementar la descripción morfológica de las especies de Dipteryx. Asimismo, otros cinco caracteres morfológicos florales fueron evaluados en siete especímenes fértiles disponibles; es así que se evaluó el color de la flor (registrado durante la colecta en campo), longitud de flor, pedicelo, cáliz y corola a tres flores maduras por espécimen.

Los especímenes fueron clasificados en grupos por dos botánicos especialistas del género Dipteryx, Haroldo de Lima y Catarina Carvalho del Jardín Botánico de Río de Janeiro, teniendo en cuenta los caracteres morfológicos de las hojas, flores y frutos. El primer grupo contiene diez especímenes de Dipteryx charapilla colectados en el departamento de Loreto, el segundo grupo contiene 38 especímenes de Dipteryx micrantha morfotipo 1 (Dm1) colectados en Loreto, Ucayali, Huánuco y Madre de Dios, y el tercer grupo contiene 23 especímenes de Dipteryx micrantha morfotipo 2 (Dm2) colectados en Loreto y Ucayali. Tres especímenes adicionales fueron agregados correspondientes a los códigos 71 y 74 que son las muestras tipo de $D$. charapilla (Schunke 362 ) y $D$. micrantha (Tessman 4967), respectivamente, y 43 que fue determinado como Dipteryx sp. (Flores 53). $D$. micrantha fue dividida en dos morfotipos que se diferencian a simple vista debido al tamaño de los foliolos y número de foliolos por hoja. El morfotipo 1 presenta foliolos grandes, elíptico-ovalados y 6-10 foliolos por hoja; y el morfotipo 2 presenta foliolos pequeños, oblongos y 8-12 foliolos por hoja.

\section{ANÁLISIS MULTIVARIADO}

Previo al análisis, se excluyeron 13 individuos con diámetro menor a $15 \mathrm{~cm}$ con el fin de enfocar en los individuos maduros con características morfológicas definidas y se realizó una prueba de correlación entre los caracteres para determinar su relación entre ellos. Posteriormente, el análisis de datos se realizó utilizando la matriz con 61 especímenes y 22 variables cuantitativas por medio de métodos multivariados de agrupamiento y de similitud usando el software PAST versión 3.1 (Hammer et al., 2001). Primero, usamos el análisis de agrupamiento a través de la media aritmética no ponderada (UPGMA) basado en la matriz de 


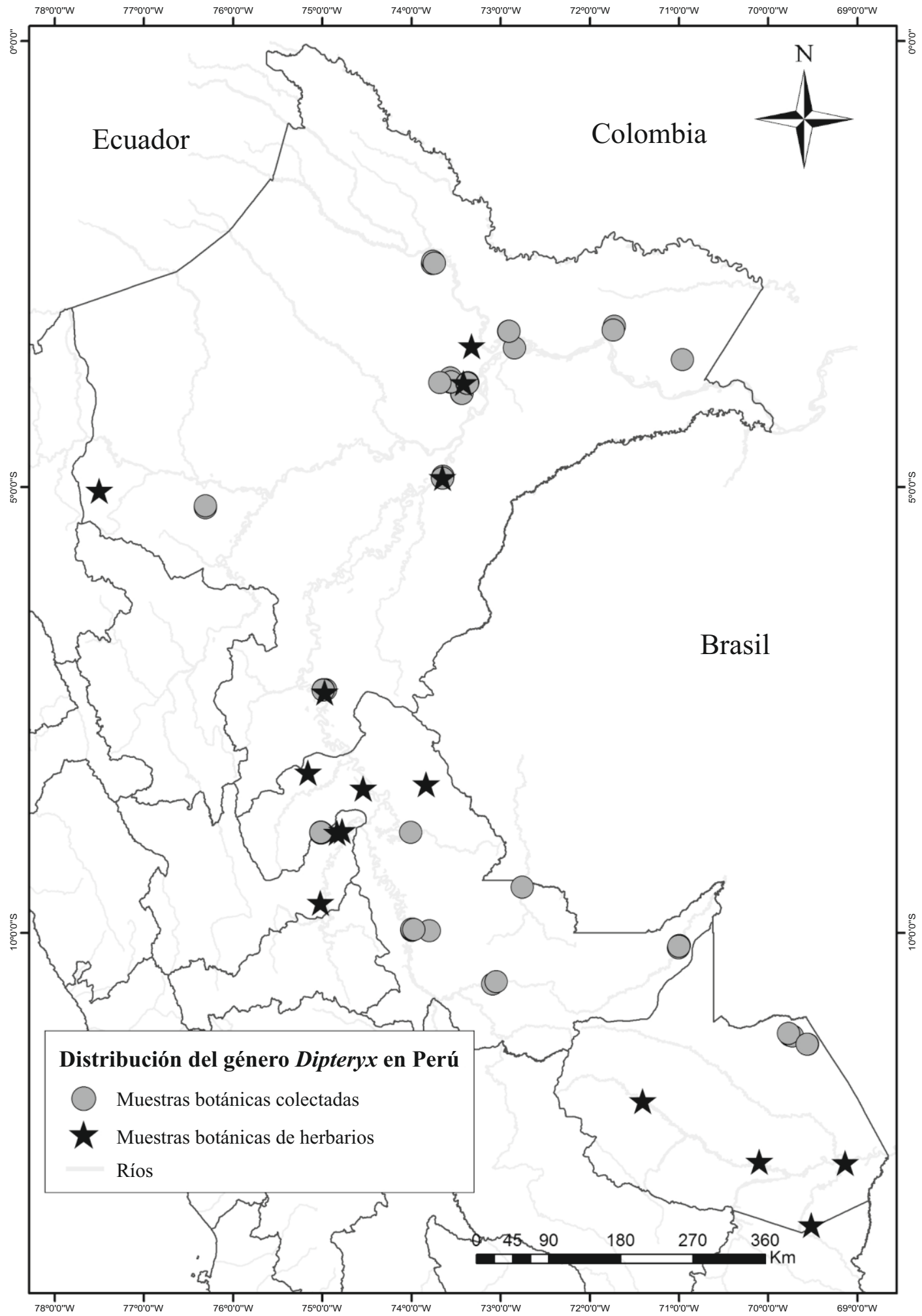

Figura 1. Distribución del género Dipteryx en la Amazonia peruana basada en las 74 muestras botánicas recopiladas. Se diferencian las 56 muestras botánicas colectadas para este estudio y las 18 muestras recopiladas de otros herbarios. 
Tabla 1. Lista de las características morfológicas evaluadas a los especímenes de Dipteryx colectados en Perú.

\begin{tabular}{|c|c|c|c|}
\hline $\mathbf{N}^{0}$ & Caracteres morfológicos & Abreviación & Unidad \\
\hline \multicolumn{4}{|c|}{ Foliares - Variables cuantitativas } \\
\hline 1 & Longitud de foliolo & LF & $\mathrm{cm}$ \\
\hline 2 & Ancho del medio del foliolo & $\mathrm{AF}$ & $\mathrm{cm}$ \\
\hline 3 & Ancho menor del tercio superior del foliolo & AS1 & $\mathrm{cm}$ \\
\hline 4 & Ancho mayor del tercio superior del foliolo & AS2 & $\mathrm{cm}$ \\
\hline 5 & Ancho menor medio del foliolo & AM1 & $\mathrm{cm}$ \\
\hline 6 & Ancho mayor medio del foliolo & AM2 & $\mathrm{cm}$ \\
\hline 7 & Ancho menor del tercio basal del foliolo & $\mathrm{AB1}$ & $\mathrm{cm}$ \\
\hline 8 & Ancho mayor del tercio basal del foliolo & AB2 & $\mathrm{cm}$ \\
\hline 9 & Extensión de base & EB & $\mathrm{cm}$ \\
\hline 10 & Ancho del peciolulo & APL & $\mathrm{cm}$ \\
\hline 11 & Longitud de peciolulo & LPL & $\mathrm{cm}$ \\
\hline 12 & Longitud de ápice & LAP & $\mathrm{cm}$ \\
\hline 13 & Longitud peciolulo /Longitud foliolo & $\mathrm{LPL} / \mathrm{LF}$ & $\mathrm{cm}$ \\
\hline 14 & Longitud / Ancho del foliolo & $\mathrm{LF} / \mathrm{AF}$ & $\mathrm{cm}$ \\
\hline 15 & Longitud de peciolo & LP & $\mathrm{cm}$ \\
\hline 16 & Longitud de raquis & LR & $\mathrm{cm}$ \\
\hline 17 & Ancho de raquis medio & AR & $\mathrm{cm}$ \\
\hline 18 & Ancho de ala medio & $A L$ & $\mathrm{~cm}$ \\
\hline 19 & Distancia promedio entre foliolos & PF & $\mathrm{cm}$ \\
\hline 20 & Número de foliolos por hoja & $\mathrm{N}$ & \\
\hline 21 & Desplazamiento vena media & (AM2-AM1) / (AB2-AB1) & \\
\hline 22 & Proporción basal/apical & $(A S 2-A S 1) /(A B 2+A B 1)$ & \\
\hline 23 & Promedio de ángulo de base & & grados \\
\hline 24 & Ancho / Longitud peciolulo & APL / LPL & \\
\hline \multicolumn{4}{|c|}{ Foliares - Variables cualitativas } \\
\hline 25 & Disposición de foliolos & & \\
\hline 26 & Forma de foliolo & & \\
\hline 27 & Base del foliolo & & \\
\hline 28 & Ápice del foliolo & & \\
\hline 29 & Borde del foliolo & & \\
\hline 30 & Patrón de nervadura & & \\
\hline \multicolumn{4}{|c|}{ Florales - Variables cualitativas y cuantitativas } \\
\hline 31 & Color & & \\
\hline 32 & Longitud total & & $\mathrm{cm}$ \\
\hline 33 & Longitud de pedicelo & & $\mathrm{cm}$ \\
\hline 34 & Longitud de cáliz & & $\mathrm{cm}$ \\
\hline 35 & Longitud de corola & & $\mathrm{cm}$ \\
\hline
\end{tabular}


disimilitud de Bray-Curtis. Segundo, realizamos los análisis de Componentes Principales (PCA) y Discriminante lineal (LDA) utilizando la misma matriz de datos. Además, se realizó el análisis de similaridad (ANOSIM; Clarke, 1993) y el análisis multivariado no paramétrico de la varianza (NPMANOVA; Anderson, 2001) para evaluar la diferencia significativa entre los grupos asignados. Finalmente, se desarrollaron mapas de distribución de las especies de Dipteryx definidas para Perú utilizando los 74 especímenes recopilados para el presente estudio.

\section{RESULTADOS}

Los análisis multivariados realizados en el presente estudio fueron consistentes y lograron diferenciar tres grupos de especies de Dipteryx. Estos grupos presentan características morfológicas particulares y distribución geográfica distintas: $D$. micrantha morfotipo 1 presenta 3 a 5 pares de foliolos, foliolos de $5.4 \mathrm{~cm} \times 11.6 \mathrm{~cm}$ en promedio, amplia distribución en la Amazonía baja, con especímenes colectados desde el sur del departamento de Loreto hasta Madre de Dios; $D$. micrantha morfotipo 2 con 4 a 6 pares de foliolos, foliolos $3.0 \mathrm{~cm} \times 7.4 \mathrm{~cm}$ en promedio, amplia distribución, con especímenes colectados en los departamentos de Loreto y Ucayali; y D. charapilla con 3 a 4 pares de foliolos, foliolos de $6.8 \mathrm{~cm}$ x 14.4 $\mathrm{cm}$ en promedio, distribución restringida a unas pocas localidades en Loreto. Estos análisis fueron realizados incluyendo 22 caracteres morfológicos y excluyendo dos variables de mayor correlación (variables 5 y 6, Tabla 1).

En el análisis de agrupamiento (UPGMA), al 0.948 de similitud, los 61 especímenes de Dipteryx se agruparon en tres grandes grupos (Figura 2a): un grupo formado por los especímenes de $D$. charapilla y la muestra tipo de la misma especie (71.DchT), un segundo grupo cercano que contiene las muestras de $D$. micrantha morfotipo 1 y una muestra de $D$. micrantha morfotipo 2 (18.Dm2), y un tercer grupo que incluye los especímenes de D. micrantha morfotipo 2 y la muestra tipo de la especie (74.DmT).

En el análisis de ordenación (PCA), los cuatro primeros componentes explican el $82.5 \%$ de la variación, obteniendo los dos primeros componentes el $47.2 \%$ y $17.4 \%$, respectivamente (Figura 2 b). En el primer componente resalta la cercanía entre los especímenes de $D$. micrantha morfotipo 1 y $D$. charapilla, y estos a su vez fueron más distantes a los especímenes de D. micrantha morfotipo 2. Los caracteres más importantes que explicaron la variación del primer componente fueron el ancho del foliolo (Valor de correlación: $r=0.97$ ) y la longitud del foliolo (0.90); mientras distancia entre foliolos (0.87), longitud del peciolulo (0.82) y número de foliolos por hoja $(-0.70)$ fueron también importantes. Longitud de raquis (0.74) y la proporción entre ancho y longitud del foliolo $(-0.74)$ fueron más importantes para el segundo componente, y extensión de base (0.87) fue importante para el tercer componente.

El análisis discriminante (LDA) define claramente los tres grupos de especies de Dipteryx; y donde el espécimen 74 correspondiente a la muestra tipo de $D$. micrantha y el espécimen 43 dejado como indeterminado fueron más similares a $D$. micrantha morfotipo 2, y el espécimen 71 correspondiente a la muestra tipo de $D$. charapilla fue más similar a los especímenes de $D$. charapilla. El primer eje discriminante es responsable por el $63.9 \%$ de la variación total, y fue determinado principalmente por el número de foliolos (Valor de varianza: -0.05) y el ancho del foliolo (0.03) (Figura 3). El segundo eje, responsable del $27.1 \%$ de la variación total, fue determinado por la longitud del peciolulo $(0.03)$; mientras el tercer eje, responsable del $4.7 \%$, fue determinado por ancho de raquis $(0.05)$ y ancho de ala medio (0.03).

Tabla 2. Análisis de la consistencia en los grupos determinados por el análisis morfométrico en muestras de Dipteryx en la Amazonia Peruana, y utilizando 10,000 permutaciones y la distancia de Bray-Curtis. Valores $\mathrm{R}$ de la prueba ANOSIM se muestran por encima de la diagonal y valores $\mathrm{F}$ de la prueba PERMANOVA se dan por debajo de la diagonal. Los números entre paréntesis son valores $p$ corregidos por Bonferroni.

\begin{tabular}{lccc}
\hline \multicolumn{1}{c}{ Grupos } & $\begin{array}{c}\text { D .micrantha } \\
\text { morfotipo 1 }\end{array}$ & $\begin{array}{c}\text { D. micrantha } \\
\text { morfotipo 2 }\end{array}$ & D. charapilla \\
\hline D. micrantha morfotipo 1 & & $0.8097(0.0003)$ & $0.5736(0.0003)$ \\
D. micrantha morfotipo 2 & $37.13(0.0003)$ & & $0.9377(0.0003)$ \\
D. charapilla & $10.26(0.0003)$ & $37.58(0.0003)$ & \\
\hline
\end{tabular}


(a) Análisis de agrupamiento (UPGMA)

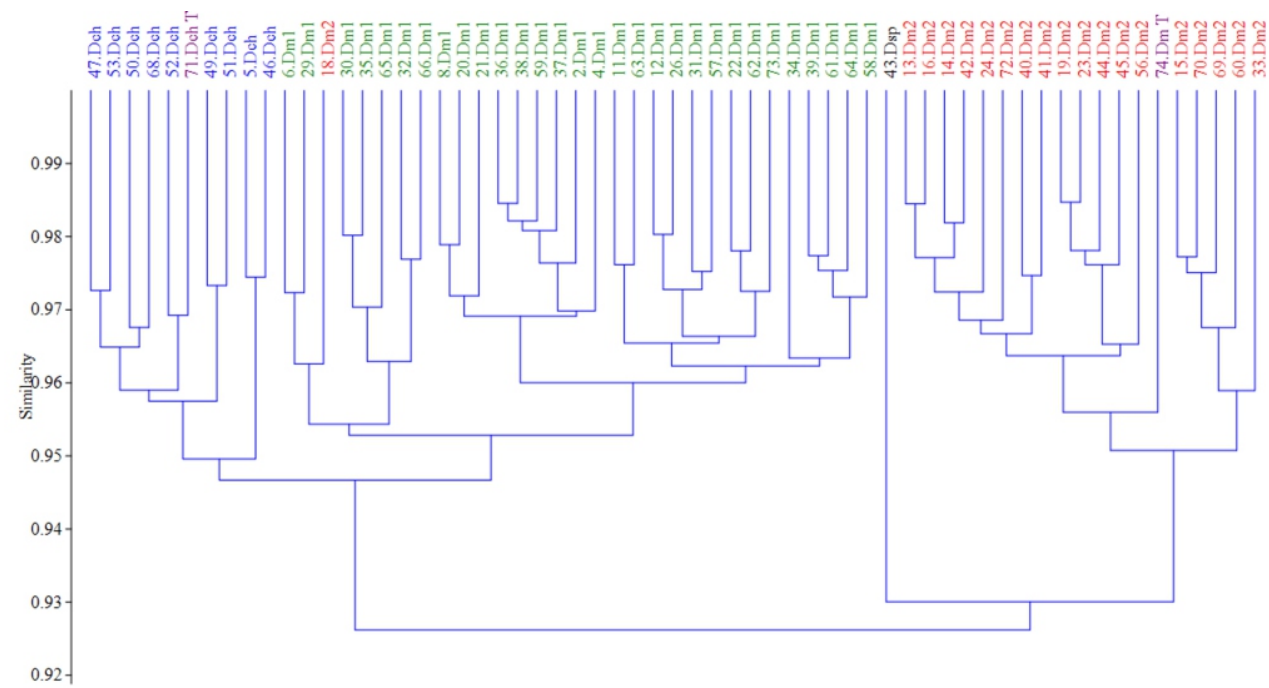

(b) Análisis de componentes principales (PCA)

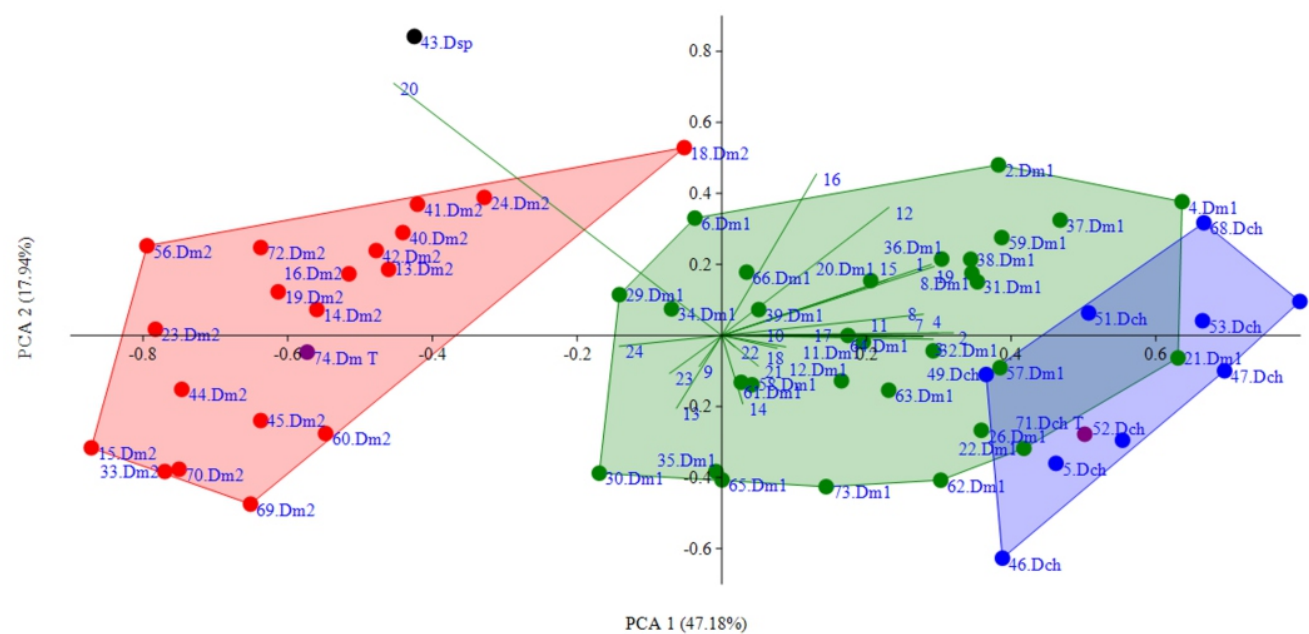

Figura 2. Análisis de agrupamiento (a) y de ordenación (b) basados en 22 caracteres morfológicos medidos en las 61 muestras de Dipteryx de Perú. Dipteryx micrantha morfotipo 1 (verde), D. micrantha morfotipo 2 (rojo) y D. charapilla (azul). Códigos 71 y 74 corresponden a muestras tipo de $D$. charapilla (Schunke 362) y $D$. micrantha (Tessman 4967), respectivamente, y 43 a un espécimen de Dipteryx sp. Los polígonos agrupan a los diferentes grupos asignados por los autores.

Los especímenes 74, 43 y 71 fueron asignados a los grupos correspondientes y corregidos para realizar los análisis de ANOSIM y PERMANOVA. Estos análisis muestran que hay diferencia significativa entre los tres grupos (Tabla 2), habiendo cierta distancia y diferencia significativa entre los dos morfotipos de D. micrantha $\left(\mathrm{R}_{\mathrm{ANOSIM}}=\right.$
$0.81, \mathrm{~F}_{\text {PERMANOVA }}=37.1$; ambos $\left.\mathrm{p}>0.001\right)$ y entre $D$. micrantha morfotipo 2 y D. charapilla $\left(\mathrm{R}_{\mathrm{ANOSIM}}=\right.$ $0.94, \mathrm{~F}_{\text {PERMANOVA }}=37.6$; ambos $\left.\mathrm{p}>0.001\right)$. Las diferencias morfológicas cuantitativas y cualitativas de los tres grupos formados se resumen en la tabla 3 utilizando los valores promedios de los 61 especímenes utilizados en el análisis. 


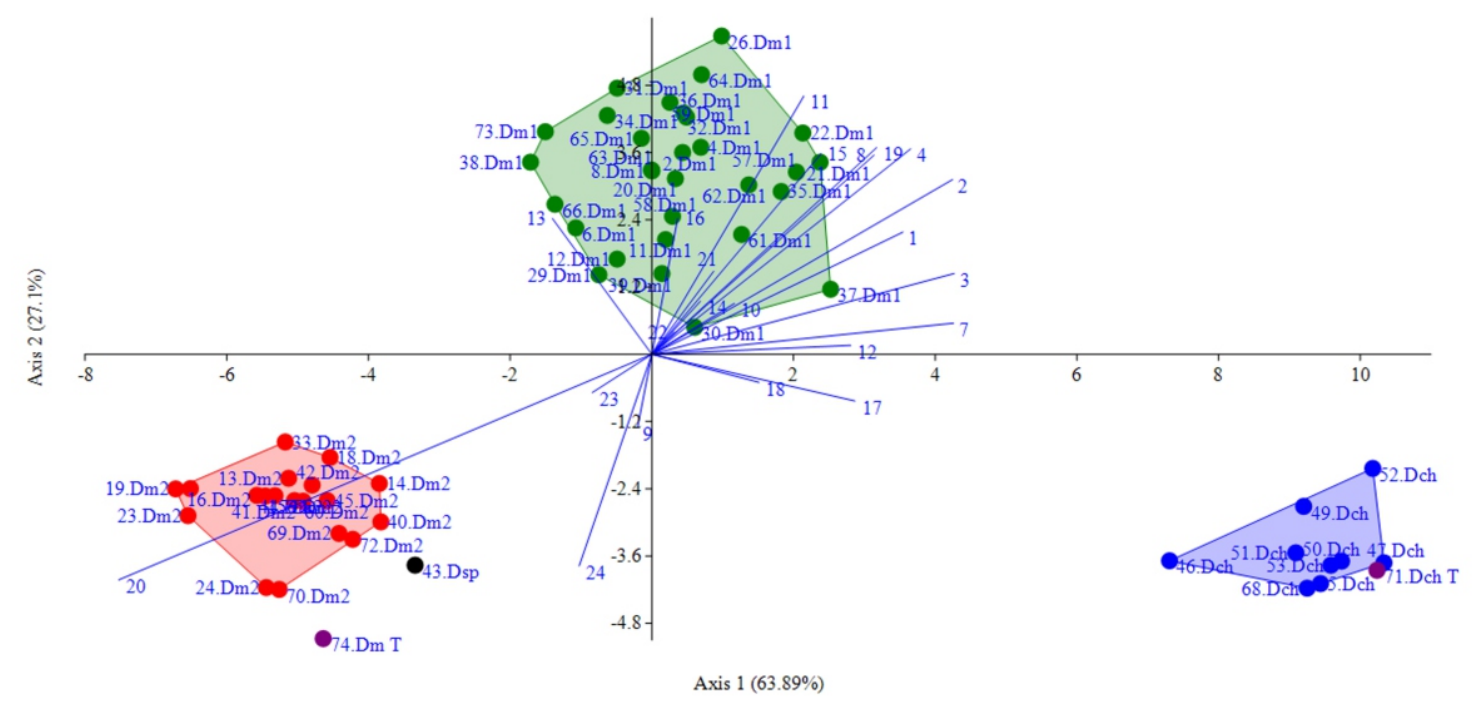

Figura 3. Gráfico de dispersión que muestra los dos primeros ejes del análisis discriminante (LDA) basado en 22 medidas morfológicas cuantitativas de especies de Dipteryx. La proyección de las variables se resalta mediante líneas azules.

\section{DISCUSIÓN}

Este estudio basado en la morfología vegetativa de los especímenes de Dipteryx del Perú muestra consistentemente la presencia de tres grupos morfológicos distintos en la Amazonía Peruana: $D$. micrantha morfotipo 1, D. micrantha morfotipo 2 y D. charapilla. Los dos morfotipos de D. micrantha podrían ser considerados como especies distintas, aunque son necesarios nuevos estudios taxonómicos incluyendo especímenes en todo el rango de distribución de la especie y colectados en otros países amazónicos. Ciertamente, existen menos especies de Dipteryx en Perú que las que se pensaba anteriormente, y así mismo demostramos que la especie de mayor comercialización es $D$. micrantha. Poder determinar los caracteres morfológicos que diferencian las especies usando análisis multivariados como en el caso de Dipteryx demuestra ser una opción viable para el estudio de otras especies maderables que presenten delimitación morfológica poco clara.

\section{CARACTERÍSTICAS MORFOLÓGICAS DE LAS ESPECIES DEDIPTERYXEN PERÚ}

Basados únicamente en la morfología, los dos morfotipos de $D$. micrantha son considerados como taxones diferentes. A simple vista, el morfotipo 1 pareciera corresponder a individuos de $D$. micrantha con características vegetativas de mayor tamaño que el morfotipo 2, sin embargo, la proporción entre los caracteres es diferente entre los morfotipos definiendo formas particulares en las hojas y en las flores. D. micrantha morfotipo 1 presenta generalmente 3 pares de foliolos elípticos $(5.4 \mathrm{~cm} \mathrm{x}$ $11.6 \mathrm{~cm}$ ) con flores de $1.3 \mathrm{~cm}$ de longitud promedio $(1.0 \mathrm{a} 1.5 \mathrm{~cm})$ y $D$. micrantha morfotipo 2 presenta 5 pares de foliolos alargados $(3.0 \mathrm{~cm} \times 7.4 \mathrm{~cm}) \mathrm{con}$ flores de $0.8 \mathrm{~cm}$ de longitud promedio $(0.6 \mathrm{a} 1.1 \mathrm{~cm})$. Más aún, pese a que la colección examinada es numerosa, es deseable desarrollar más estudios enfocados específicamente en reflejar la variación morfológica intraindividual, incluyendo la que se observa a lo largo del año.

Los dos morfotipos de $D$. micrantha difieren considerablemente de $D$. charapilla que presenta generalmente 3 pares de foliolos ovalados $(6.8 \mathrm{~cm} \mathrm{x}$ $14.4 \mathrm{~cm}$ ) con flores de 2.2 a $3.0 \mathrm{~cm}$ de longitud. Los caracteres morfológicos evaluados en los especímenes recopilados en el presente estudio no coinciden con las descripciones originales de $D$. alata o $D$. odorata, correspondiendo potencialmente estos nombres a determinaciones equívocas en especímenes colectados en Perú. D. alata podría confundirse vegetativamente con $D$. micrantha morfotipo 2 porque ambas especies presentan foliolos alargados. Sin embargo, los especímenes de D. alata revisados en los herbarios virtuales presentan 6 a más pares de foliolos y frutos globosos (4 $\mathrm{cm}$ x $5 \mathrm{~cm})$, mientras $D$. micrantha morfotipo 2 
presenta 4-6 pares de foliolos y frutos oblongos (3 $\mathrm{cm} \times 5 \mathrm{~cm}$ ). Además, los lugares de colecta sugieren que estas especies prefieren condiciones ecológicas diferentes; mientras $D$. alata ocurre en bosques de savanna del sureste de Brazil y Bolivia, $D$. micrantha ocurre en los bosques húmedos amazónicos (Barham, 2005).

En el caso de $D$. odorata, esta especie podría fácilmente confundirse con $D$. charapilla debido a que ambas especies presentan 6-8 foliolos, foliolos coriáceos y basales alternos, sin embargo, $D$. charapilla se diferencia por la base redonda de los foliolos, ápice cuspidado y flores más grandes que las presentes en $D$. odorata que cuenta con base marcadamente asimétrica y ápice acuminado (Willdenow, 1802). La cercanía en la descripción morfológica de $D$. charapilla con $D$. rosea reportada para Brasil sugiere potencialmente la presencia de sinonimia entre estas especies aunque Macbride hace notar que son especies diferentes debido al tamaño de las flores (Macbride, 1943). En el caso de $D$. ferrea, esta especie es considerada actualmente un nombre sinónimo de $D$. micrantha.
Cabe resaltar que existe variación en el tamaño de los foliolos de las especies de Dipteryx reportadas en Perú. Más aún los individuos pequeños, con diámetro menor a $15 \mathrm{~cm}$, presentan mayor variación en las variables medidas como la longitud y ancho del foliolo. Los individuos pequeños, que se encuentran bajo el dosel o en estado juvenil presentan mayor tamaño foliar que los individuos que se encuentran sobre el dosel o los arboles senescentes, siendo estos eliminados antes de realizar los análisis para evitar el efecto de edad y disponibilidad de luz en los resultados (Morales et al., 1991; Puntiei et al., 2001; Louman, 2001; Raven, et al., 1992). La importancia de las flores y frutos en estudios taxonómicos han sido de mucha ayuda al identificar especies de Dipteryx que se encuentran cercanamente emparentadas (Mcbride, 1943; Francisco, 2010). Es así, que en este estudio se realizó el seguimiento de algunos individuos en campo para poder obtener la descripción morfológica de las flores y los frutos que resultaron ser marcadamente diferentes entre las especies.

Tabla 3. Descripción morfológica de las especies basada en las mediciones de 61 especímenes de Dipteryx colectados en Perú. Se muestran los valores promedios y los valores máximos y mínimos en paréntesis.

\begin{tabular}{|c|c|c|c|}
\hline Carácter & $\begin{array}{l}\text { D. micrantha morfotipo } 1 \\
\text { (Dm1) }\end{array}$ & $\begin{array}{l}\text { D. micrantha morfotipo } 2 \\
\text { (Dm2) }\end{array}$ & D. charapilla \\
\hline Foliolo & Elíptico-ovalado & Oblongo-alargado / Oblongo-elíptico & Ovalado \\
\hline Nervadura del foliolo & Eucamptódroma & Eucamptódroma & Elíptico-ovalado / Eucamptodroma \\
\hline Borde del foliolo & Entero & Entero & Entero \\
\hline Base del foliolo & Redonda a obtusa & Redonda a cordada & Redonda \\
\hline Ápice del foliolo & Ampliamente acuminado & Ampliamente acuminado & Cuspidado \\
\hline Foliolos basales & Opuestos & Opuestos & Alternos \\
\hline Número de foliolos & $7(6-10)$ & $10(8-12)$ & $6(5-8)$ \\
\hline Longitud de foliolo & $11.6 \mathrm{~cm}(6.3-22.4 \mathrm{~cm})$ & $7.4 \mathrm{~cm}(4.5-13.1 \mathrm{~cm})$ & $14.4 \mathrm{~cm}(8.9-21.3 \mathrm{~cm})$ \\
\hline Ancho de foliolo & $5.4 \mathrm{~cm}(3.4-8.0 \mathrm{~cm})$ & $3 \mathrm{~cm}(2.2-4.6 \mathrm{~cm})$ & $6.8 \mathrm{~cm}(4.9-8.5 \mathrm{~cm})$ \\
\hline Longitud de peciolulo & $0.6 \mathrm{~cm}(0.4-0.8 \mathrm{~cm})$ & $0.3 \mathrm{~cm}(0.2-0.5 \mathrm{~cm})$ & $0.5 \mathrm{~cm}(0.4-0.6 \mathrm{~cm})$ \\
\hline Ancho de peciolulo & $0.2 \mathrm{~cm}(0.1-0.3 \mathrm{~cm})$ & $0.2 \mathrm{~cm}(0.1-0.2 \mathrm{~cm})$ & $0.2 \mathrm{~cm}(0.1-0.3 \mathrm{~cm})$ \\
\hline Distancia entre foliolos & $4.0 \mathrm{~cm}(2.0-6.8 \mathrm{~cm})$ & $2.3 \mathrm{~cm}(1.2-4.2 \mathrm{~cm})$ & $4.2 \mathrm{~cm}(1.8-6.5 \mathrm{~cm})$ \\
\hline Longitud del raquis & $13.3 \mathrm{~cm}(5.7-22.8 \mathrm{~cm})$ & $10.9 \mathrm{~cm}(5.2-19.1 \mathrm{~cm})$ & $10.8(5.5-15.2 \mathrm{~cm})$ \\
\hline Color de la flor & Lilas & Rosadas & Fucsia \\
\hline Longitud de flor & $1.3 \mathrm{~cm}(1.0-1.5 \mathrm{~cm})$ & $0.8 \mathrm{~cm}(0.6-1.1 \mathrm{~cm})$ & $2.7 \mathrm{~cm}(2.2-3.0 \mathrm{~cm})$ \\
\hline Longitud de pedicelo & $0.2 \mathrm{~cm}(0.2-0.3 \mathrm{~cm})$ & $0.1 \mathrm{~cm}(0.1-0.2 \mathrm{~cm})$ & $0.5 \mathrm{~cm}(0.4-0.6 \mathrm{~cm})$ \\
\hline Longitud de cáliz & $0.2 \mathrm{~cm}(0.2-0.3 \mathrm{~cm})$ & $0.2 \mathrm{~cm}(0.1-0.2 \mathrm{~cm})$ & $0.4 \mathrm{~cm}(0.2-0.6 \mathrm{~cm})$ \\
\hline Longitud de corola & $0.9 \mathrm{~cm}(0.6-1.0 \mathrm{~cm})$ & $0.5 \mathrm{~cm}(0.3-0.7 \mathrm{~cm})$ & $1.7 \mathrm{~cm}(1.4-1.9 \mathrm{~cm})$ \\
\hline
\end{tabular}


Dipteryx micrantha morfotipo 1

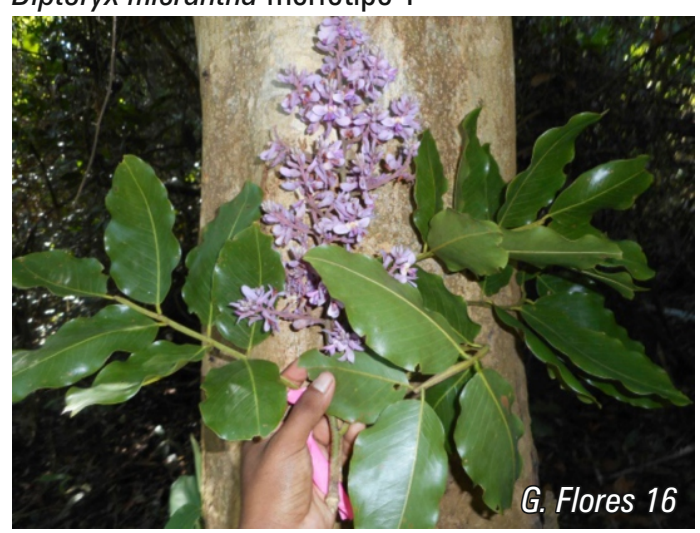

\section{Dipteryx micrantha morfotipo 2}

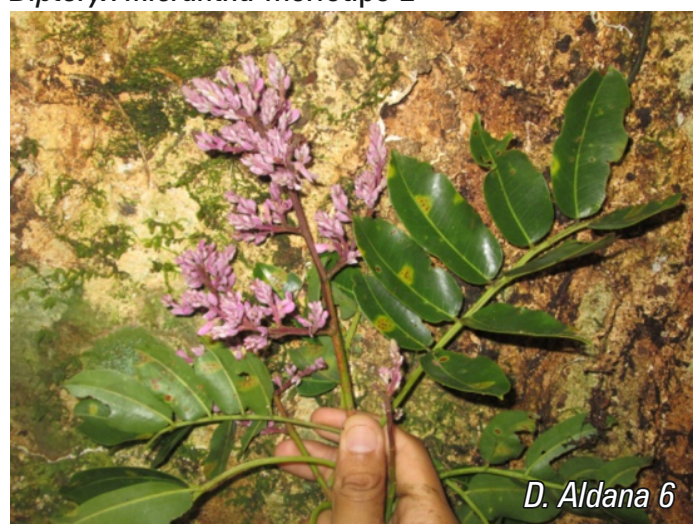

\section{Dipteryx charapilla}

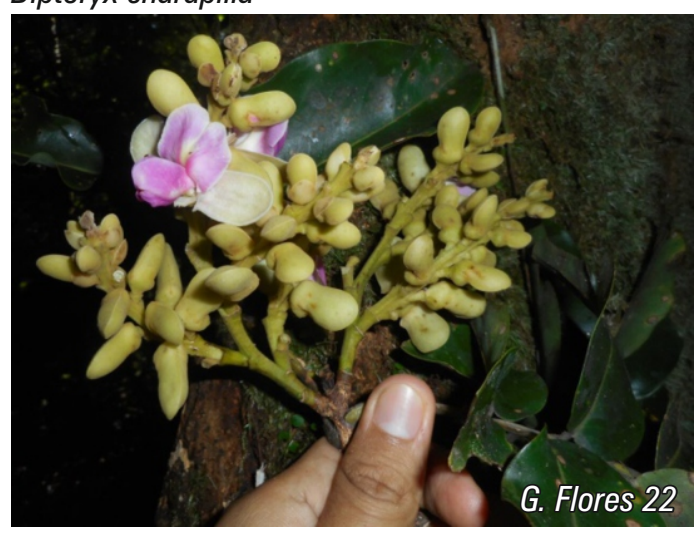

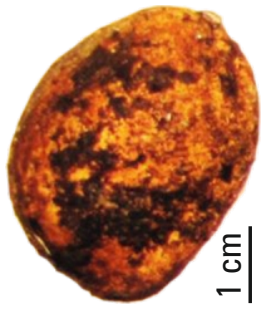

G. Hidalgo s.n.
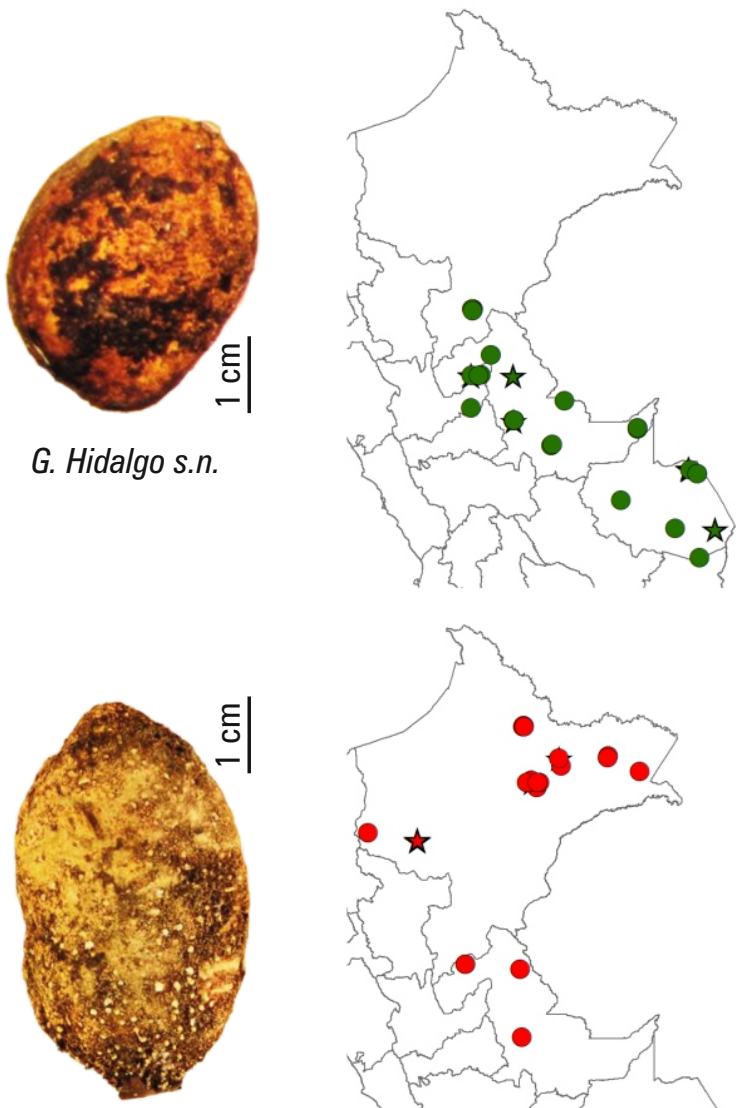

G. Hidalgo s.n.
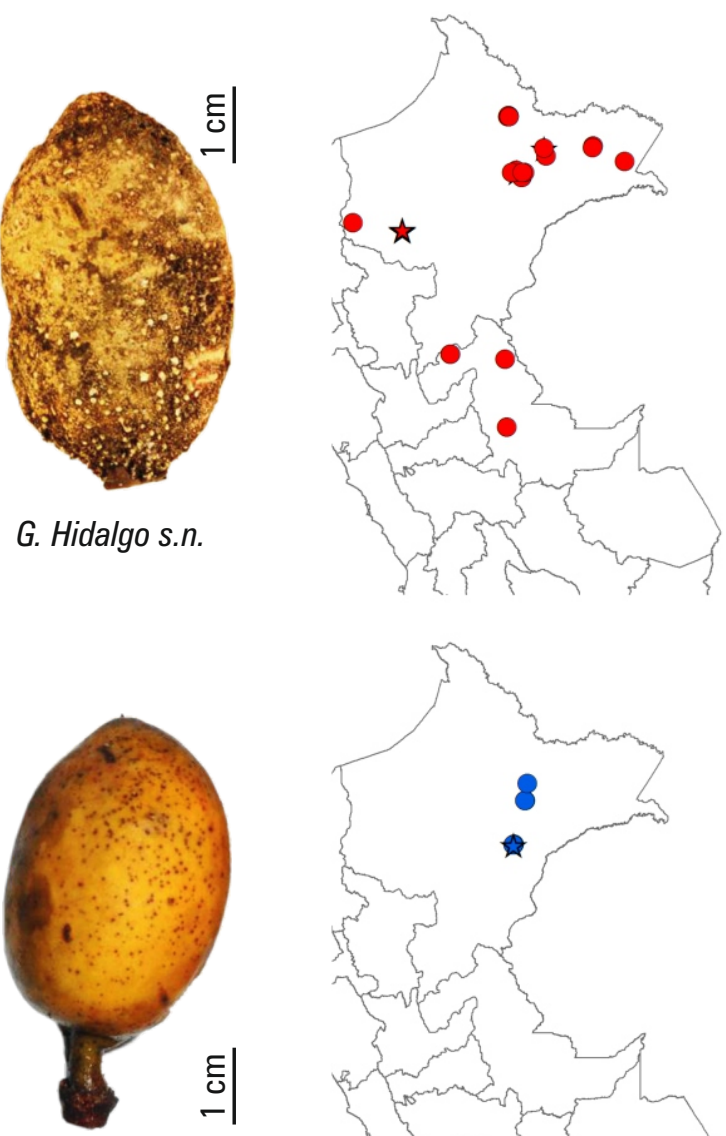

G. Flores 173

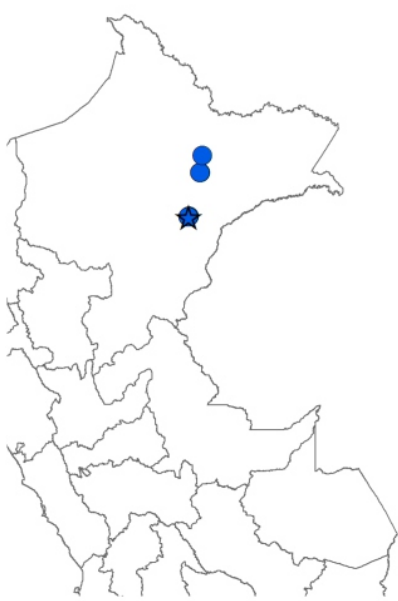

Figura 4. Morfología y distribución geográfica de las especies de Dipteryx en Perú. Las estrellas en los mapas representan los especímenes con diámetro menor a $15 \mathrm{~cm}$ que no fueron utilizados en los análisis multivariados. 


\section{DISTRIBUCIÓN GEOGRÁFICA DE LAS ESPECIES}

Los resultados muestran que las especies de Dipteryx en Perú tienen distribución geográfica distinta (Figura 4). D. micrantha morfotipo 1 tiene amplia distribución y ocurre en los departamentos de Loreto (zona sur), Ucayali, Madre de Dios y Huánuco; D. micrantha morfotipo 2 ocurre en Loreto y Ucayali; y $D$. charapilla está restringida al departamento de Loreto donde existen reportes en las localidades de Jenaro Herrera, Puerto Almendras y Río Mazán. Cabe resaltar que las tres especies de Dipteryx ocurren en el departamento de Loreto, siendo este departamento la región más diversa para el género. Fuera de Perú, $D$. micrantha también ocurre en Bolivia, Brasil, Colombia, y Ecuador. Mientras $D$. charapilla solo presenta reportes para Perú, especímenes similares determinados bajo el nombre de $D$. rosea fueron también reportados en Brasil y Venezuela.

El conocimiento de la delimitación de las especies de Dipteryx y la distribución geográfica de las mismas contribuirá significativamente a los estudios genéticos que el Instituto de Investigaciones de la Amazonía Peruana viene realizando con el fin de determinar marcadores genéticos para la identificación de las especies forestales maderables y la verificación genética de la madera en la región amazónica. En particular, este presente estudio es la base de los estudios que venimos realizando en la relación filogenética de las especies de Dipteryx y la caracterización de las poblaciones de Dipteryx micrantha en la Amazonía Peruana.

\section{ESPECIE DE MAYOR EXPORTACIÓN}

Por mucho tiempo se ha creído que Dipteryx odorata es la especie del género Dipteryx con mayor extracción y exportación del Perú, es así que el Ministerio de Agricultura incluye en la lista de especies maderables a Dipteryx odorata y Coumarouna odorata, bajo los nombres comunes de charapilla y shihuahuaco, respectivamente. Sin embargo, la revisión exhaustiva de más de 70 especímenes de herbario demuestra que $D$. odorata y $C$. odorata no han sido aún reportadas en Perú y potencialmente, estos nombres son erróneamente utilizados para nombrar los especímenes de $D$. charapilla (Brako \& Zarucchi, 1993; Spichiger et al., 1989) o a nivel comercial a los dos morfotipos de D. micrantha. Basándose en los volúmenes extraídos a nivel de departamento, podemos indicar que $D$. micrantha morfotipo 1 es probablemente la especie de mayor extracción en el Perú, proveniente de los departamentos de Ucayali y Madre de Dios con volúmenes de 37 mil metros cúbicos y 125 mil $\mathrm{m}^{3}$, respectivamente (MINAGRI, 2014). La extracción en Loreto es también alta $\left(51 \mathrm{mil} \mathrm{m}^{3}\right)$ y probablemente corresponde a la extracción de ambos morfotipos de D. micrantha. Otros departamentos registrados con extracción de madera de Dipteryx son Huánuco $\left(1100 \mathrm{~m}^{3}\right)$, Junín $\left(71 \mathrm{~m}^{3}\right)$ y San Martín (34 $\left.\mathrm{m}^{3}\right)$ (MINAGRI, 2014).

Poder contar con estudios ecológicos de las especies de Dipteryx es una prioridad para el sector forestal, en particular estudios relacionados a la tasa de crecimiento de los árboles, edad de madurez sexual entre otros factores que determinan los ciclos de corta idóneos para el adecuado manejo de estas especies. Asimismo, es importante asegurar la permanencia de poblaciones naturales en diferentes partes del país para poder asegurar la conservación de la diversidad genética de las especies y la adaptación a los cambios ambientales presentes y futuros. Por lo tanto, inventarios de las tres especies de Dipteryx descritas en el presente estudio deberían ser prioridad en áreas de conservación para asegurar la conservación de poblaciones naturales suficientes en bosques primarios no intervenidos.

\section{AGRADECIMIENTOS}

Los autores agradecen al Programa Nacional de Innovación para la Competitividad y Productividad (Innóvate Perú), por el financiamiento bajo contrato 381-PNICP-PIAP-2014, y al Instituto Thünen, Alemania a través del Proyecto Large Scale Project on Timber Verification por su colaboración científica. De igual manera, también agradecen al Servicio Nacional Forestal y de Fauna Silvestre (SERFOR) y al Servicio Nacional de Áreas Naturales Protegidas por el Estado (SERNANP) por la amplia colaboración prestada para la ejecución del trabajo de campo y por los permisos de investigación para realizar las colectas del material biológico (R.D.G. N 060-2015-SERFOR-DGGSPFFS, R.D. $\mathrm{N}^{\circ}$ 001-A-2015-SERFOR-DGGSPFFS-DGSPF, R.D. $N^{\circ}$ 001-2016-SERNANP-DGANP). Agradecemos también a T.R. Baker por sus comentarios durante la elaboración del manuscrito y a R.T. Pennington por la revisión del mismo.

\section{BIBLIOGRAFÍA CITADA}

Anderson, M. J. 2001. A new method for nonparametric multivariate analysis of variance. Austral Ecology, 26: 32-46.

Barham, J. 2005. Tribe Dipterygeae. In: Lewis G., Schrire B., Mackinder B., Lock M., eds. Legumes of the world. Kew: Royal Botanic Gardens, 250-251. 
Brako, L.; Zarucchi, J. 1993. Catálogo de las Angiospermas y Gimnospermas del Perú. Monographs in Systematic Botany from the Missouri Botanical Garden, 45: 1-1286.

Cardoso, D; São-Mateus, WMB; da Cruz, DT; Zartman, CE; Komura, DL; Kite, G; Prenner, G; Wieringa, JJ; Clark, A; Lewis, G; Pennington, RT; de Queiroz, LP. 2015. Filling in the gaps of the papilionoid legume phylogeny: The enigmatic Amazonian genus Petaladenium is a new branch of the early-diverging Amburaneae clade. Molecular Phylogenetics and Evolution, 84: 112-124.

Clarke, K.R. 1993. Non-parametric multivariate analysis of changes in community structure. Australian Journal of Ecology, 18: 117-143.

Dávila, N., Honorio, E., Salazar, A. 2008. Fichas de identificación de especies maderables de Loreto, Perú. Instituto de Investigaciones de la Amazonía Peruana. Proyecto Focal Bosques. Iquitos, Perú. $30 \mathrm{p}$.

de Lima, H.C., 1989. A Checklist of the Dipterygeae species. Manuscript doc.

Degen, B.; Ward, S. E.; Lemes, M. R.; Navarro, C.; Cavers, S.; Sebbenn, A. M. 2013. Verifying the geographic origin of mahogany (Swietenia macrophylla King) with DNA-fingerprints. Forensic Science International: Genetics, 7(1): 55-62.

Ducke, W.A. 1949. Notas sobre a Flora Neotropica-II: As Leguminosas da Amazonia Brasileira (ed. 2). Bol. Tecn. Inst. Agron. 18: $1-248$.

Francisco, V. 2010. Filogenia molecular y morfológica de la tribu Dipterygeae (Papilionideae, Leguminosae). Tesis de maestría. Instituto de Pesquisas Jardín Botánico de Rio de Janeiro. Escuela Nacional de Botánica tropical. Rio de Janeiro, Brasil. 77 p.

Hammer, ̌̌.; Harper, D.A.T.; Ryan, P.D. 2001. PAST: Paleontological statistics software package for education and data analysis. Palaeontologia Electronica, 4(1): 1-9. http://palaeo-electronica.org/2001_1/past/ issue 1_01.htm

Louman, B., D Quirós y M Nilsson. (eds). 2001. Silvicultura de bosques latifoliados húmedos con énfasis en América Central. Serie técnica, manual técnico no. 46. CATIE, Turrialba, Costa Rica. 265 p.

Mcbride, J. F. 1943. Leguminosae. 13(3/1): 3-507. In: J. F. Macbride (ed.) Flora of Peru. Publ. Field Museum Natural History, Bot. Ser. Field Museum, Chicago. 507 p.
Martínez-Cabrera, D.; Zavala-Chávez, F.; Terrazas, T. 2011. Estudio morfométrico de Quercus sartorii y $Q$. xalapensis (Fagaceae). Revista mexicana de biodiversidad, 82(2): 551-568.

Mascarenhas Souza, I.; Silveira Funch, L.; Paganucci de Queiroz, L. 2014. Morphological analyses suggest a new taxonomic circumscription for Hymenaea courbaril L. (Leguminosae, Caesalpinioideae). PhytoKeys, 38: 101-118.

MINAGRI, 2014. Perú Forestal en Números 2014. Ministerio de Agricultura y Riego. Lima, Perú. $204 \mathrm{p}$.

Monteagudo, A.; Huamán, M. 2010. Catálogo de los árboles y afines de la Selva Central del Perú. Arnaldoa, 17(2): 203-242.

Monteagudo, A.; Valenzuela, L.; Vásquez, R.; Gonzáles, R. D. P. R.; Phillips, O. L.; LopezGonzalez, G. 2015. Primer catálogo de los árboles y afines de la Reserva Comunal El Sira, Perú. Arnaldoa, 21(1): 127-164.

Morales, D., Jimenez, S., Wagner, J., Larcher, W. 1992. Caracterización morfológica e histológica de las hojas de sol y sombra de Persea indica (L) Spreng. y Persea americana Mill. Vieraea, 21: 61-76.

Puntieri, J.G., Damascos, M.A., Souza, M.S. 2001. Tendencias ontogenéticas en el tamaño y la forma de las hojas de Nothofagus pumilio (Poepp. et Endl.) Krasser (Fagaceae). Ecología Austral 11(2): 105-114.

Putzel, L. 2010. The tree that held up the forest: Shihuahuaco (Dipteryx spp.) and the Chinese timber trade. Tesis de doctorado. City University of New York, Estados Unidos de Norteamérica. $156 \mathrm{p}$.

Raven, P.; Everet, R.; Eichhorn, S. 1992. Biología de las plantas. Volumen 2. Barcelona. Editorial Reverté. 402p.

Reynel, C.; Pennington, R.; Pennington, T.; Flores, C.C.; Daza. A. 2003. Arboles útiles de la Amazonia peruana y sus usos, un manual con apuntes de identificación, ecología y propagación de las especies. Herbario de la Facultad de Ciencias Forestales de la Universidad Nacional Agraria-La Molina, Lima. $509 \mathrm{p}$.

Schneider, C. A.; Rasband, W. S.; Eliceiri, K. W. 2012. NIH Image to ImageJ: 25 years of image analysis. Natmethods, 9(7): 671-675.

Soladoye, M. O.; Sonibare, M. A.; Chukwuma, E. C. 2010. Morphometric study of the genus Indigofera Linn.(Leguminosae-Papilionoideae) in South-Western Nigeria. International Journal of Botany, 6(3): 343-350. 
FOLIA

Amazónica

ANÁLISIS MORFOMÉTRICO DE LAS ESPECIES

DE DIPTERYX EN LA AMAZONÍA PERUANA

Spichiger, R.; Méroz, J.; Loizeau, P.; Stutz de Ortega, L. 1989. Contribución a la flora de la Amazonia Peruana. Los arboles del Arboretum Jenaro Herrera; v. 1: Moraceae a Leguminosae. Brossiera, 43: 1-358.

Vásquez, R. 1997. Flórula de las reservas biológicas de Iquitos, Perú. Monographs in Systematic Botany 63. Missouri Botanical Garden, USA. 1046 p.
Willdenow, C. 1802. Species Plantarum. Editio quarta, 3(2): 910

Recibido: 3 de Junio del 2016

Aceptado para publicación: 15 de Octubre del 2016 


\section{ANEXO 1. DESCRIPCIÓN DE LAS ESPECIES DE DIPTERYX DE PERÚ}

\section{Dipteryx micrantha Harms "morfotipo 1", Fig. 5}

Árboles dominantes de hasta $40 \mathrm{~m}$ de altura y $2 \mathrm{~m}$ de diámetro. Corteza externa lenticelada, color marrón claro, naranja a rojo oscuro cuando el individuo es adulto, color crema a verdusco cuando es juvenil, presenta escamas de ritidoma que desprenden aisladamente dejando huellas impresas ("martillado"). Ramita terminal lenticelada. Hojas compuestas alternas en espiral, imparipinnadas, $3-5$ pares de foliolos, distanciados de 2.0 a $6.8 \mathrm{~cm}$, foliolos basales opuestos, elíptico-ovalados, base redonda a obtusa, ápice ampliamente acuminado, $6.3-22.4 \mathrm{~cm}$ de largo y 3.4-8.0 $\mathrm{cm}$ de ancho. Inflorescencias en panículas terminales, flores 1.0 a $1.5 \mathrm{~cm}$ de longitud, pedicelo de $0.2 \mathrm{a} 0.3 \mathrm{~cm}$ de longitud, cáliz de 0.2 a $0.3 \mathrm{~cm}$ de longitud, corola de 0.6 a $1.0 \mathrm{~cm}$ de longitud, receptáculo y sépalos lilas, alas y quilla violetas, estandarte lila con machas violetas. Frutos drupas indehiscentes, carnosas, de forma globosa y una semilla alargada.

Especímenes revisados: PERU. Dpto. Huánuco, Dist. Honoria, 0851'31"S 7446'26"W, 30 de Marzo 1965, E. Jensen $22 \mathrm{fl}$. (MOL), 0852'46"S 07449'58"W, 5 de Diciembre 1964, E. Jensen 144 fl. (MOL), Dist. Yuyapichis, Dantas, 09³9'57"S 75¹'12"W, 16 de Noviembre 1988, Kroll 101 fl. (MOL), Dpto. Loreto, Dist. Contamana,

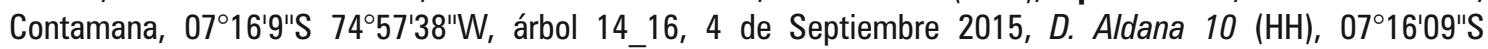
7459'13"W, árbol 14_28, 5 de Septiembre 2015, D. Aldana 11 (HH), 07¹6'27"S 7459'15"W, árbol 14_31, 5 de Septiembre 2015, D. Aldana 12 (HH), 07²18'28"S 7458'19"W, 2 de Noviembre 1982, C. Reynel 808 fl. (MOLL), Dpto.

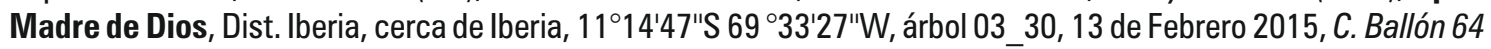
(HH), 11¹4'47"S $69{ }^{\circ} 33^{\prime} 40^{\circ} W$, árbol 04_02, 14 de Febrero 2015, C. Ballón 65 (HH), Dist. Iñapari, Concesión


árbol 03_28, 9 de Septiembre 2015, G. Flores $6(\overline{\mathrm{HH}}), 1^{\circ} 07^{\prime} 25^{\prime \prime} \mathrm{S} 69^{\circ} 46^{\prime} 07^{\prime \prime} \mathrm{W}$, árbol 03_30, 11 de Septiembre 2015, G. Flores 8 (HH), Dist. Manu, Estación Los Amigos, 12³4'06"S 7005'47"W, 10 de Diciembre 2003, A. Maceda \& F.

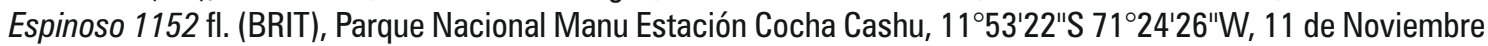
1988, R. Foster 5520 (MOL), Dist. Tambopata, Cuzco Amazónico, 12³4'58"S 697'58"W, 14 de Diciembre 1989, $A$. Genty 68734 (MOL), Dist. ?, Parque Nacional Bahuaja Sonene, 13¹6'45"S 69³0'41"W, 16 de Noviembre 1997, C. Diaz \& H. Ramirez 9529 (MOL), Dpto. Ucayali, Dist. Iparia, Concesión forestal “Oxigeno para el Mundo", 08`52'12"S $74^{\circ} 00^{\prime} 35^{\prime \prime}$, árbol 24 13, 13 de Diciembre 2015, G. Flores 38 (HH), Dist. Irazola, Macuya, 0852'34"S 7500'13"W, árbol 05 05, 19 de Julio 2015, E. Mejía 1 (HH), 0852'46"S 7500'48"W, árbol 05_17, 22 de Julio 2015, E. Mejía 6 (HH), 0852'21"S 7500'32"W, árbol 05_30, 30 de Julio 2015, E. Mejía 8 (HH), 0851'55"S 7501'01"W, árbol 05 32, 30 de Julio 2015, E. Mejía 10 (HH), 08`52'14"S 7500'58"W, árbol 05_33, 30 de Julio 2015, E. Mejía 11 (HH), Dist.

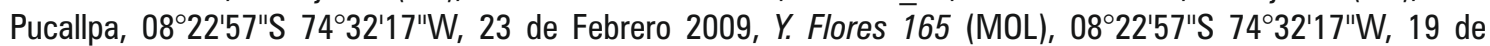

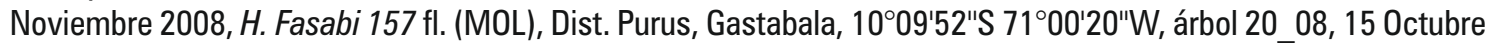
2015, L. Huaratapiro $127 \mathrm{fl}$. (HH), 1008'47"S 7159'58"W, árbol 20_19, 16 Octubre 2015, L. Huaratapiro 130 (HH), 1008'16"S 7059'50"W, árbol 20 23, 16 Octubre 2015, L. Huaratapiro 132 (HH), Dist. Raymondi, San Juan de Inuya, 10³4'09"S 7305'11"W, árbol 22_34, 18 de Noviembre 2015, G. Flores $34(\mathrm{HH}), 10^{\circ} 32^{\prime} 47^{\prime \prime} \mathrm{S} 73^{\circ} 03^{\prime} 00^{\prime \prime} \mathrm{W}$, árbol 22 09, 5 de Noviembre 2015, G. Flores 29 (HH), Dist. Tahuania, Santa Clara, 0958'03"S 7359'43"W, árbol 21_21, 24 de Octubre 2015, G. Flores 26 (HH), 0957'50"S 7359'38"W, árbol 21_06, 22 de Octubre 2015, G. Flores 15 (HH), 0957'50"S 7359'22"W, 21_09, 22 de 0ctubre 2015, G. Flores $17(\mathrm{HH}), \overline{09}^{\circ} 57^{\prime} 24^{\prime \prime} \mathrm{S} 73^{\circ} 59^{\prime} 32^{\prime \prime} \mathrm{W}, 21$ 08, 22 de Octubre 2015, G. Flores 16 fl. (HH), 0957'27"S 7358'58"W, árbol 21_13, 23 de Octubre 2015, G. Flores 21 (HH), 0957'17"S 7359'33"W, árbol 21_03, 22 de Octubre 2015, G. Flores 13 (HH), 0957'38"S 7358'23"W, árbol 21 16, 23 de Octubre 2015, G. Flores $23(\mathrm{HH})$, Dist. Yurua, Santa Rosa, 09² $29^{\prime} 06^{\prime \prime} S 072^{\circ} 45^{\prime} 22^{\prime \prime}$, árbol 18_32, $2 \overline{1}$ de Febrero 2016, G. Hidalgo 23 (HH), Dist. Pucallpa, 08²2'57"S 74³2'17"W, 8 de Abril 1993, NN 95 (MOL).

\section{Dipteryx micrantha Harms "morfotipo 2", Fig. 6}

Árboles de 30-35 m de altura y hasta $1.30 \mathrm{~m}$ de diámetro. Corteza externa lenticelada, color marrón claro, naranja a rojo oscuro cuando el individuo es adulto, color crema a verdusco cuando es juvenil, presenta escamas de ritidoma que desprenden aisladamente dejando huellas impresas ("martillado"). Ramita terminal lenticelada. Hojas compuestas alternas en espiral, imparipinnadas, 4-6 pares de foliolos, distanciados de 1.2 a $4.2 \mathrm{~cm}$, foliolos basales opuestos, oblongo alargados a oblongo elípticos, base redonda a cordada, ápice ampliamente acuminado, 4.5-13.1 
$\mathrm{cm}$ de largo y 2.2-4.6 cm de ancho. Inflorescencias en panículas terminales, flores 0.6 a $1.1 \mathrm{~cm}$ de longitud, pedicelo de 0.1 a $0.2 \mathrm{~cm}$ de longitud, cáliz de 0.1 a $0.2 \mathrm{~cm}$ de longitud, corola de 0.3 a $0.7 \mathrm{~cm}$ de longitud, receptáculo verde claro, sépalos rosados claros, alas, quilla y estandarte rosadas. Frutos drupas indehiscentes, carnosas, de forma oblonga y una semilla alargada.

Especímenes revisados: PERU. Dpto. Loreto, Dist. Indiana, Sucusari, $03^{\circ} 15^{\prime} 00^{\prime \prime S} 72^{\circ} 54^{\prime} 30^{\prime \prime} \mathrm{W}$, árbol 13_01, 8 de Julio 2015, G. Hidalgo $14(\mathrm{HH}), 3^{\circ} 15^{\prime} 05^{\prime \prime} S$ 7254'16"W, árbol 13_02, 14 de Julio 2015, G. Hidalgo 15 (HH),

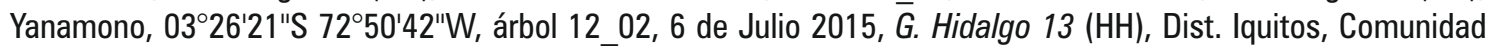

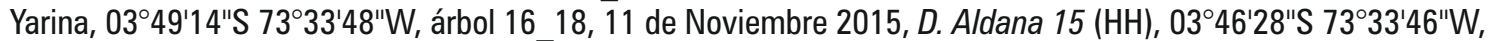
árbol 16 11, 18 de Noviembre 2015, D. $\bar{A}$ Aldana $16(\mathrm{HH}), 03^{\circ} 49^{\prime} 10^{\prime \prime} \mathrm{S} 73^{\circ} 33^{\prime} 17^{\prime \prime}$, árbol 16 24, 23 de Noviembre

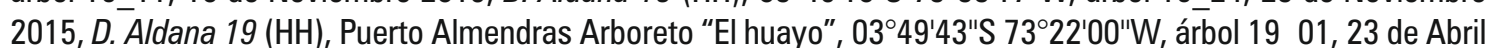
2016, G. Flores $50(\mathrm{HH}), 03^{\circ} 49^{\prime} 43^{\prime \prime} \mathrm{S} 73^{\circ} 40^{\prime} 55^{\prime \prime}$, árbol 19 02, 23 de Abril 2016, G. Flores 51 (HH), Dist. Jeberos, Jeberos, $05^{\circ} 13^{\prime} 50^{\prime \prime} \mathrm{S} 76^{\circ} 18^{\prime} 35^{\prime \prime} \mathrm{W}$, árbol 35_05, 9 de Julio 2016, G. Flores 55 (HH), 05¹2'28"S 76¹8'27"W, árbol 35 01, 9 de Julio 2016, G. Flores 56 (HH), Dist. Manseriche, Rio Marañon, 05²'36"S 77³0'00"W, 20 de Enero 1925, G. Tessmann $4967 \mathrm{fl}$. (F), Dist. Napo, Comunidad Huiririma, 02²9'28"S 7345'55"W, árbol 11 01, 18 de Julio 2015, D. Aldana 2 fl. (HH), $02^{\circ} 28^{\prime} 44^{\prime \prime} \mathrm{S} 73^{\circ} 44^{\prime} 44^{\prime \prime} \mathrm{W}$, árbol 1130,18 de Julio 2015, D. Aldana $\overline{6}$ fl. (HH), 02²8'04"S

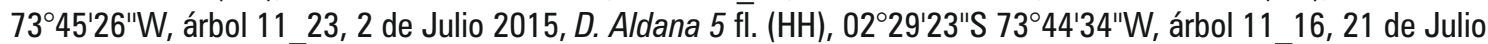
2015, D. Aldana 4 fl. $(\overline{\mathrm{H}} \mathrm{H})$, Dist. Pevas, Pevas, 03¹1'41"S 7143'17"W, árbol 30 01, 7 de Mayo 20̄16, G. Flores 52

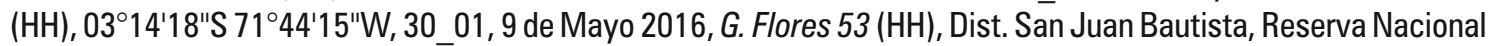
Allpahuayo-Mishana, 0356'51"S 73²6'08"W, árbol 01_01, 7 de Noviembre 2014, C. Ballón 29 (HH), árbol 01 01,

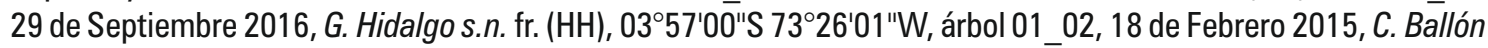
31 (HH), 0349'59"S 73²4'59"W, 16 de Febrero 1987, A. Gentry 55993 fl. (RB), Dist. San Pablo, Comunidad Unión Progresista, 03³4'08"S 7057'31"W, árbol 33_01, 3 de Junio 2016, G. Flores 54 (HH), Dpto. Ucayali, Dist.

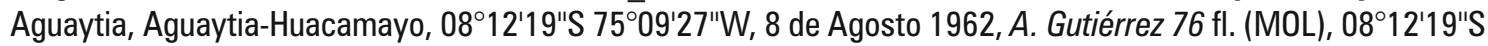
$75^{\circ} 9^{\prime} 27^{\prime \prime} W, 20$ de Febrero 1962, A. Gutiérrez 40 fl. (MOL), Dist. Irazola, Reserva von Humboldt, 08¹9'58"S 7349'58"W, 27 de Enero 2001, T. Pennington 17090 fl. (MOL), Dist. Tahunia, Comunidad Bolognesi, 0958'22"S 734'ㄴ'

\section{Dipteryx charapilla (J.F. Macbr.) Ducke, Fig. 7}

Árboles de 20-30 m de altura y hasta $65 \mathrm{~cm}$ de diámetro. Corteza externa lenticelada, color crema a grisáceo en individuos adultos y color verdusco cuando el individuo es juvenil, presenta escamas de ritidoma que desprenden aisladamente dejando huellas impresas ("martillado"). Ramita terminal lenticelada. Hojas compuestas alternas en espiral, imparipinnadas, 3-4 pares de foliolos, distanciados de 3 a $6 \mathrm{~cm}$, foliolos basales alternos, elíptico-ovalados, base redonda, ápice cuspidado, 8.9-21.3 cm de largo y $4.9-8.5 \mathrm{~cm}$ de ancho. Inflorescencias en panículas terminales, flores de 2.2 a $3.0 \mathrm{~cm}$ de longitud, pedicelo de 0.4 a $0.6 \mathrm{~cm}$ de longitud, cáliz de 0.2 a $0.6 \mathrm{~cm}$ de longitud, corola de 1.4 a $1.9 \mathrm{~cm}$ de longitud, sépalos amarillos claros, alas, quilla y estandarte fucsias. Frutos drupas indehiscentes, carnosas, de forma elíptica y una semilla alargada.

Especímenes revisados: PERU. Dpto. Loreto, Dist. Jenaro Herrera, Centro de Investigaciones Jenaro Herrera, 045'ㄴ'4"S 73³8'59"W, árbol 02_01, 14 de Agosto 2015, D. Aldana $22(\mathrm{HH})$, árbol 02_01, 10 de Julio 2016, D. Aldana $32 \mathrm{fl}$. (HH), árbol 02_01, 24 Septiembre 2016, G. Hidalgo 131 fr. (HH), 0452'44"S 7338'51"W, arbol 02 02,

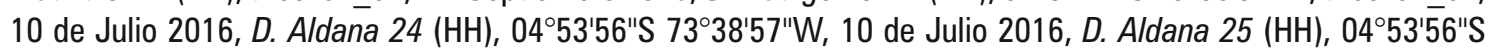
73³8'57.3"W, arbol 02 04, 10 de Julio 2016, D. Aldana 26 (HH), 0454'00"S 73³9'00"W, 25 de octubre 2002, T. Pennington 1138 (MOL), Dist. San Juan Bautista, Puerto Almendras Arboreto "El Huayo", 0349'59"S 73²2'25"W, árbol 19 01, 16 de Julio 2016, D. Aldana $27 \mathrm{fl}$., 0349'59"S 73²2'25"W, árbol 19 01, 10 de Diciembre 2016, G.

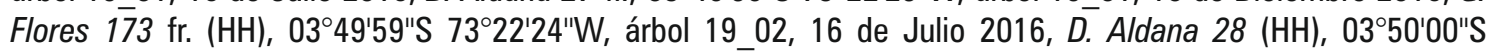
$73^{\circ} 22$ '24"W, árbol 19_03, 16 de Julio 2016, D. Aldana 29 (HH), 0350'00"S 73²2'25"W, árbol 19_04, 16 de Julio 2016, D. Aldana $30(\mathrm{HH}), 03^{\circ} 50^{\prime} 00^{\prime \prime} \mathrm{S} 73^{\circ} 22$ '24"W, árbol 19 05, 16 de Julio 2016, D. Aldana 31 (HH), Dist. Mazán, Río Mazán, 04²5'13"S 73¹9'22"W, 10 de Marzo 1935, J. Shunke 362 fl. (US). 




Figura 5. Dipteryx micrantha "morfotipo 1". A. ramita con hoja y flores. B. Flor. C. Estandarte. D. Ala externa. E. Ala interna. F. Pistilo. G. Contorno general del fruto; B-F superpuestos en una cuadrícula de $0.5 \mathrm{~cm}$ de lado. (Especimen con flores, L. Huaratapiro 127, HH; fruto, G. Hidalgo s.n. árbol 18 24). 


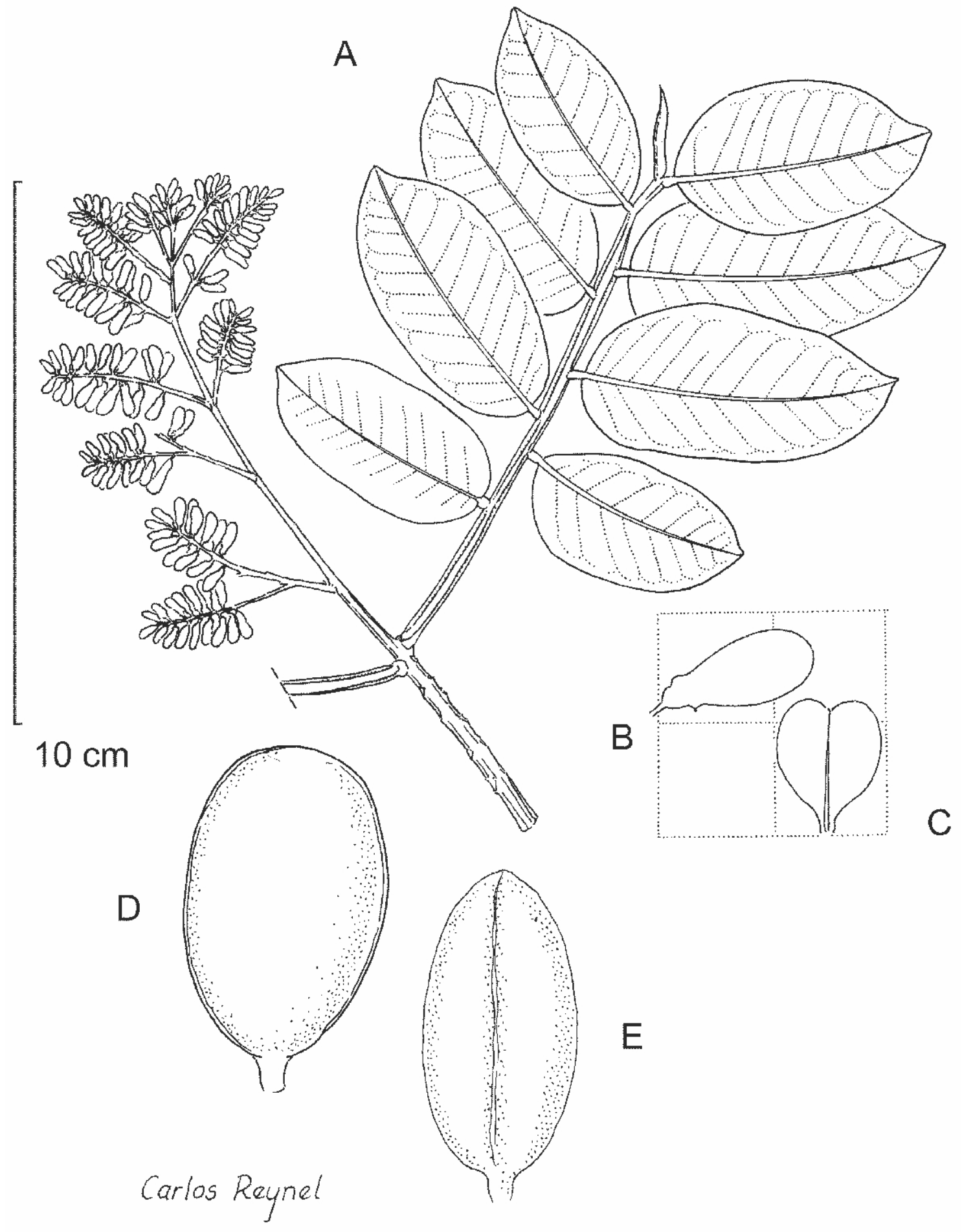

Figura 6. Dipteryx micrantha "morfotipo 2". A. Ramita con hoja y flores. B. Flor. C. Estandarte. D. Ala externa. E. Ala interna. F. Pistilo. G. Fruto; B-F superpuestos en una cuadrícula de $0.5 \mathrm{~cm}$ de lado. (Especimen con flores, D. Aldana 5, HH; fruto, G. Hidalgo s.n. árbol 01_01). 


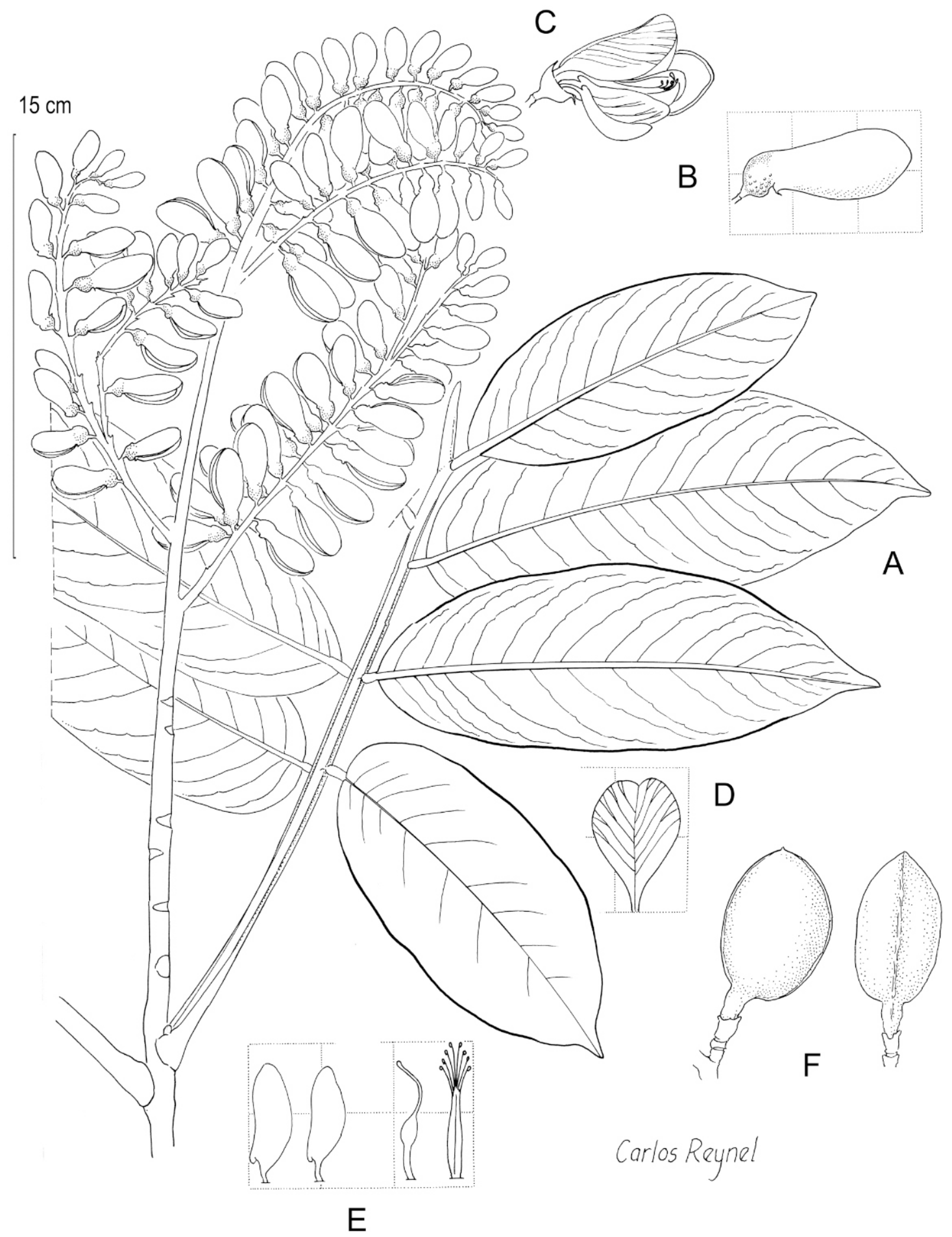

Figura 7. Dipteryx charapilla. A. ramita con hoja y flores. B. Flor, superpuesta en una cuadrícula de $1 \mathrm{~cm}$ de lado. C. Flor, con la mitad más próxima del cáliz retirada. D. Estandarte, superpuesto en una cuadrícula de $1 \mathrm{~cm}$ de lado. E. De izquierda a derecha, ala externa, ala interna, pistilo y androceo, el último visto de arriba; todos superpuestos en una cuadrícula de $1 \mathrm{~cm}$ de lado. $\mathbf{F}$. Fruto, vista de frente y lateral. (Especimen con flores, D. Aldana 27, HH; Especimen con frutos, $G$. Flores $173, \mathrm{HH})$. 